\title{
Salmonellosis, aspectos clínicos y epidemiológicos
}

DRA. VAIERIA PRADO J. ${ }^{*} y^{* *}$.

\section{INTRODUCCION}

Las Salmonellas son bacterias que pertenecen a la Familia Enterobacteriáceas y todas ellas son patógenas provocando cuadros que afectan principalmente al intestino tanto de hombres como de animales.

El conocimiento de este grupo de gérmenes y su relación con enfermedades humanas y animales, se remonta al siglo pasado, ya que fueron aislados por primera vez por Smith y Salmon en 1885 en cerdos muertos de peste porcina y al primer representante de este género individualizado se le denominó Salmonella cholera-suis.

Desde entonces hasta nuestros días se han llegado a identificar cerca de 900 especies diferentes de Salmonellas y cada día se agregan nuevos serotipos a la ya larga lista.

Las salmonellosis constituyen en nuestro país, un problema médico importante y año a año consumen gran parte de los recursos que se destinan para recuperación de la salud.

Es la población infantil la que sufre el principal embate de estos gérmenes. Por esta razón nos parece importante crear conciencia acerca del problema, conocerlo en todas sus dimensiones para lograr un mejor control de él.

Creemos necesario aclarar que el término salmonellosis se refiere a todos los cuadros clínicos provocados por Salmonellas en el hombre, lo que incluye además de Tifoidea, Intoxicaciones alimentarias, Diarrea Aguda, Septicemias, Meningitis, Artritis, Osteomelitis (Tabla No 1).

Desde el punto de vista de los agentes en

\footnotetext{
* Departamento Medicina Experimental, Facultad de Medicina, Sede Santiago Oriente, Lniversidad de Chile.

** Hospital Luis Calvo Mackenna.
}

C U A D R O N $: 1$

Cuadros clinicos provocado por Salmonella en el hombre

1.- Tifoidea (septicemias clínicas)

2.- Septicemias

3.- Intoxicación alimentaria

4.- Diarrea aguda (infección enteral)

5.- Procesos supurados secundarios: moningitis, osteoartritis, osteomielitis.

juego en los distintos cuadros, la infección por Salmonella en el hombre debe ser considerada bajo 2 aspectos:

- Salmonellas propias del hombre: S. Typhi, S. paratyphi.

- Salmonellas originales de los animales: entre nosotros las más frecuentes: $\mathrm{S}$. typhimurium, S. newington, S. bareilly, S. london (Tabla 2).

El segundo grupo cada día adquiere mayor importancia clínica y creemos no equivocarnos al asegurar que constituyen la zoonosis más frecuente en Chile, aun cuando las reales dimensiones del problema en nuestro medio no está totalmente estudiado, ya que la notificación es muy deficiente y se estima que alcanza sólo al 1\% de los casos reales que se producen. 
T A B L A N N 2

Clasificación de las salmonellas de acuerdo a especie parasitada

1.- Salmonellas patógenas del hombre:

S. typhi

S. paratyphi (Para A).

2.- Salmonellas patógenas de los animales y hombre:

S. schothmüllerii

S. typhimurium

S. newington

S. enteritidis

3.- Salmonellas patógenas de los animales:

S. ovis

S. anatum

S. pullorum, etc...

\section{FUENTES}

Se ha llegado a establecer que las Salmonellas se encuentran en una gran variedad de animales desde elefantes a peces, como comensales en la mayoría de ellos. Un porcentaje importante de vacunos, ovinos, porcinos y aves de corral están infectados por ellas, animales que constituyen la principal fuente de alimentación protéica del hombre.

Parece lógico pensar que si aumenta la incidencia de Salmonellosis en estos animales, seguirán aumentando las salmonellosis humanas, mientras no se efectúe un control adecuado de las técnicas de crianza y alimentación de estos animales.

En nuestro país un estudio realizado en cerdos aparentemente sanos por el Dr. Darío Pinto (1) que efectuó cultivos de ganglios mesentéricos de cerdos faenados en los mataderos de Santiago, demostró que un $11 \%$ de ellos estaban infectados con Salmonellas, entre ellas: S. typhimurium, S. reading, $S$. newington, $S$. stanley en ese mismo orden de frecuencia.

Otro estudio similar realizado en gatos por R. Aburto (1965) (2), en nuestro país demostró que un $75 \%$ de estos animales domésticos estaban infectados con Salmonellas, entre ellas la más frecuente S. typhimurium ( $43 \%$ de las cepas) y en segundo lugar S. Schottmullerii, lo que muestra que estos animales tan frecuentes en nuestra población pueden jugar un papel importante en la transmisión de las salmonellosis.

\section{TRANSMISION}

Los mecanismos de transmisión son variados:

- animal al hombre por carnes infectadas o alimentos contaminados con deposición animal.

- hombre a hombre por alimentos contaminados con deposición humana y mediante fomites.

Es importante en este circuito destacar el papel de los alimentos en la transmisión, ya sea alimentos infectados, principalmente carnes infectadas que se consumen insuficientemente cocinadas o alimentos contaminados durante su preparación y que no son preservados en forma adecuada.

Las Salmonellas son destruidas por efecto del calor pero alimentos ya cocinados, pueden fácilmente contaminarse por el contacto con alimentos crudos, en la superficie de trabajo o mediante los utensilios de cocina y en el ambiente de calor y humedad de la cocina se dan las condiciones ideales para la multiplicación de estos gérmenes.

Luego el alto índice de infección por Salmonella traduce la alta contaminación fecal de nuestro ambiente. Un ejemplo de la amplia diseminación de salmonellas en nuestro ambiente, lo encontramos en los resultados de una investigación realizada en el río $\mathrm{Ma}$ pocho por Castillo(3), en la cual se encontró Salmonellas en el $41,7 \%$ de las muestras de agua recogida de dicho río, aislándose $\mathrm{S}$. schottmullerii, S. typhimurium, S. derby, S. stanley y $S$. bareilly en ese orden de frecuencia.

En lo que se refiere a infección humana los datos estadísticos de que disponemos indican que en los últimos 10 años se notifican alrededor de 5.000 casos de Tifoidea al año y se estima similar la cifra de Salmonellas aisladas de casos de diarrea aguda, septicemias o localizaciones articulares en niños, aunque en estos cuadros la notificación es aún más deficiente.

Un estudio de anticuerpos antisalmonellas efectuado en población adulta e infantil del Area Oriente de Santiago durante 1973 (4) mostró que un 55,8\% de las personas estudiadas, todas ellas aparentemente sanas habían tenido contacto anterior con Salmonella. El análisis de este estudio serológico, en relación a frecuencia de especie de Salmonella infectante demostró que las especies de Salmonella más frecuente en nuestra población son: S. typhi, S. london, S. 
T A B L A N $: 3$

Distribución de 560 personas con anticuerpos antisalmonellas según frecuencia de especie infectante

\begin{tabular}{lcr}
\hline $\begin{array}{l}\text { Especie de } \\
\text { salmonella }\end{array}$ & $\begin{array}{c}\text { No personas con } \\
\text { anticuerpos }\end{array}$ & $\%$ \\
\hline Salmonella typhi & 340 & 29,4 \\
Salmonella london & 295 & 25,5 \\
Salmonella newington & 197 & 17,0 \\
Salmonella typhimurium & 180 & 15,5 \\
Salmonella schottmullerii & 78 & 6,7 \\
Salmonella hirschfeldii & 65 & 5,9 \\
\hline T O T A L & $1.155^{*}$ & 100,0 \\
\hline
\end{tabular}

* Este total es mayor que el número de personas infectadas ya que algunas de ellas habían tomado contacto con más de una especie de salmonella.

\section{T A B L A N N 4}

Distribución según localización de 456 cepas de Salmonellas aisladas en Hosp. S.J. de Dios años 1972 - 1974

\begin{tabular}{lc}
\hline & No cepas \\
\hline Deposición & 426 \\
Sangre & 27 \\
Orina & 1 \\
Pus articular & 1 \\
Pus ocular & 1 \\
Pus pleural & 1 \\
\hline T O T A L & 457 \\
\hline
\end{tabular}

\section{T A B L A $\mathrm{N}: 5$}

Distribución segun especie de 457 cepas de Salmonella aisladas en Hosp. S.J. de Dios años 1972 - 1974

\begin{tabular}{lc}
\hline Especie & No cepas \\
\hline S. typhimurium & 399 \\
S. newington & 45 \\
S. enteritidis & 4 \\
S. grupo E & 3 \\
S. london & 2 \\
S. derby & 2 \\
S. bareilly & 1 \\
S. hirschfeldii & 1 \\
\hline T O T A L & 457 \\
\hline
\end{tabular}

Distribución según especie de 441 cepas de Salmonellas aisladas en Hospital L. Calvo Mackenna año 72-73-74

\begin{tabular}{lr}
\hline S. typhimurium & 186 \\
S. typhi & 114 \\
S. bareilly & 26 \\
S. newington & 67 \\
S. schottmullerii & 14 \\
S. paratyphi & 9 \\
S. enteritidis & 6 \\
S. stanly & 6 \\
S. hirschfeldii & 5 \\
S. grupo C & 1 \\
S. grupo B & 4 \\
S. london & 2 \\
S. eastbourne & 1 \\
\hline T O T A L & 441 \\
\hline
\end{tabular}

newington y S. typhimurium en este orden de frecuencia (Tabla $\mathrm{N}^{\circ} 3$ ).

Se observan claras diferencias en el poder patógeno de las distintas especies y así aunque Salmonella london es la segunda en frecuencia después de Salmonella typhi en el estudio serológico, sólo muy ocasionalmente se aisla de enfermos, lo cual estaría indicando que la infección por Salmonella london cursaría como una enfermedad subclínica o inaparente en la mayoría de los casos.

En cambio, S. typhimurium provoca diarrea aguda en lactantes que en el $50 \%$ de los casos se acompaña de síndrome disentérico, con evoluciones clínicas tormentosas la mayoría de las veces, siendo cuadros difíciles de manejar desde el punto de vista terapéutico y con tendencia a generalizarse la infección determinando septicemias (5).

Las siguientes tablas nos muestran la cuantía y distribución por especie de las Salmonellas aisladas de pacientes pediátricos en los últimos 3 años en 2 hospitales de Santiago.

Tablas N.os 4 y 5 Hospital San Juan de Dios.

Tablas Nos. 6 y 7 Hospital Luis Calvo Mackenna.

Estas tablas nos muestran que las Salmonellas originales de los animales son las que producen más problemas en pediatría, siendo la secuencia lógica que la madre portadora contamine a su hijo lactante al manipular los alimentos con técnicas higiénicosanitarias deficientes. 
T A B L A N $: 7$

Distribución según localización de 441 cepas de Salmonellas aisladas en Hospital

L. Calvo Mackenna año 72-73-74

\begin{tabular}{lr}
\hline Deposicion & 224 \\
Sangre & 188 \\
Orina & 12 \\
Pus pleural & 3 \\
Pus articular & 1 \\
Pus & 12 \\
Ambiente pabellón & 1 \\
\hline T O T A L & 441 \\
\hline
\end{tabular}

\section{ENFOQUE TERAPEUTICO}

En lo que se refiere al manejo terapéutico de las Salmonellas, el criterio varía según la localización.

- Cuando la Salmonella, especialmente las pertenecientes al grupo que constituye zoonosis, se encuentran sólo al nivel enteral, determinando diarreas o gastroenteritis, existe hoy en día consenso unánime, después de muchos estudios bien controlados in vivo, que no deben usarse antibióticos ya que aunque in vitro las cepas muestran sensibilidad frente a determinado quimioterápico, al administrarlo en pacientes, no sólo prolonga el período de excreción de la Salmonella sino que en ocasiones, se agrava el cuadro. Por esto nuestra conducta frente a infección enteral por Salmonella es no dar antibióticos $(6,7)$.

- Cuando la Salmonella está provocando septicemia o focos secundarios: meningitis, artritis; también hay consenso en que se debe usar antibiótico y éste será el de elección frente a cada especie de Salmonella.

Para finalizar quisiéramos hacer un llamado a nuestros colegas pedíatras para que nos ayuden a descubrir la parte sumergida del iceberg que constituyen las salmonellosis, que piensen en estos agentes, los busquen y en lo posible se invesguen las fuentes de infección.

En este último aspecto nos parece indispensable efectuar un mejor control de los animales que constituyen alimento para el hombre.

\section{REFERENCIAS}

9.- Pinto D. 1965.- Investigación de Salmonella en ganglios mesentéricos de cerdos aparentemente normales. Tesis Fac. Ciencias Pec. y Mcdic. Veterinaria, U. de Chile.

2.- Aburto, $R, 1965$ - Investigación de Salmonella en el gato. Tesis Fac. Cicncias Pec. y Med. Veterinaria, U. de Chile.

3.- Castillo, G., Cordano, A.M. 1973.- Contaminación por Enterobacteriaceae del río Mapocho y canal Las Mercedes. Comunicación personal.

4.- Prado, V. y col.- Indice de infección por Salmonella en población del Area Oriente de Stgo. Rev. Méd. Chile. 102: 9. 1974.

5.- Prado, V., Donoso, E., Zilleruelo, G.- Infección enteral por Salmonella typhimurium en lactante y la sensibilidad in vitro de este germen patógeno a 7 antibióticos. Rev. Chil. Ped. 42: 91, 1971.

6.- Rossertein, B.- Salmonellosis in infants and childrens. J. of Ped. 701: 1, 1957.

7.- Aserkoff, B., Bennett, J.- Effect of Therapy in Acute Salmonellosis on Salmonellac in Feces. J. New Engl. J. Med. 281, 636, 1969.

8.- KAK Rowland.- Typhoid fever and other Salmonella infections. Brit J. Hosp. Med. Jan. 1974.

9.- Cordano, A.M., Virgilio, R.- Diversos serotipos de Salmonella encontrados en cl país desde 1945. Bol. Inst. Bact. Chile, Vol. 15-64, 1973. 